\title{
The changing treatment of arthritis
}

\author{
Leslie G. Cleland, Director of Rheumatology, Susanna M. Proudman, Visiting \\ Rheumatologist, Michael J. James, Chief Hospital Scientist, and Peter Penglis, \\ Dawes Scholar, Rheumatology Unit, Royal Adelaide Hospital, Adelaide
}

Index words: anti-inflammatory drugs, osteoarthritis, rheumatoid arthritis, COX inhibitors.

(Aust Prescr 2000;23:26-7)

Arthritis falls into two very broad categories, which are not mutually exclusive. The most common is osteoarthritis, in which a primary feature is degeneration of articular cartilage, often accompanied by evidence of soft tissue inflammation ranging from subtle to overt. The other broad category contains inflammatory arthropathies, of which rheumatoid arthritis and psoriatic arthritis are the most common examples.

There is no evidence to suggest that medications have a significant influence on the natural history of osteoarthritis. Management centres on diagnosis and qualified reassurance regarding the generally slow tempo of osteoarthritis. (This contrasts with the debilitating effects and destructive potential of inflammatory arthropathies.) Patients with osteoarthritis are advised to remain physically active without abusing affected joints unnecessarily. Exercise prescriptions should be designed to enhance and maintain general fitness and not focus narrowly on the affected joint. ${ }^{1}$ Physiotherapists can provide advice about exercises for general fitness and for maintaining the strength and range of movement in affected joints.

Medication can be used to provide relief from pain. It may be taken strategically before an activity which the patient particularly wishes to maintain but is known to cause

\section{In this issue...}

Australian prescribers will be hearing a lot about the COX-2 inhibitors in the next few months. While Peter Brooks informs us how these drugs work, Les Cleland and his colleagues remind us that anti-inflammatory drugs are not the only treatment of arthritis.

While new drugs are usually widely available, blood products can be in short supply. Mark Dean explains why more donors are needed to help prevent haemolytic disease of the newborn. With winter approaching it is timely for Peter Wormald to review the treatment of sinusitis. The cooler weather also prompts the appearance of deathcap mushrooms in parts of Australia.

Infestations can occur at any time of year and Chris Commens tells us how to manage patients with scabies. discomfort. Paracetamol is the recommended first-line drug for pain relief in osteoarthritis. This recommendation is based on its greater safety compared to conventional non-steroidal anti-inflammatory drugs (NSAIDs).

NSAIDs are recommended for second-line analgesia on an 'as required' basis, with an accompanying warning about the increased risk for potentially catastrophic gastrointestinal adverse effects. While the relative risk for major upper gastrointestinal bleeding is similar at all ages, the absolute risk becomes far greater in the elderly. Indeed, the prevalence of osteoarthritis in the elderly is so high (affecting most people over 60 years of age) and the risk of upper gastrointestinal events from NSAIDs (which generally result in hospitalisation and sometimes death) is so substantial, that these unwanted effects are a major public health problem.

How will this situation change with the introduction of selective cyclo-oxygenase-2 (COX-2) inhibitors? We can expect a significant reduction in hospitalisation and death from NSAIDrelated upper gastrointestinal events. However, given the demographics of NSAID use, what new risks might emerge? A major unresolved question is the impact that COX-2 inhibitors may have on the risk of thrombotic vascular events. This risk is unlikely to be seen in short-term efficacy trials or early postmarketing surveillance, but may only be identified in longerterm epidemiological studies. To date, there is no clinical evidence that such a risk exists. However, the selectivity of COX-2 inhibitors gives some reasons for concern.

While COX-1 is the isoenzyme of COX involved in the protective homeostasis of the stomach, where its inhibition is generally undesirable, this isoenzyme is also responsible for the production of thromboxane $\mathrm{A}_{2}$ by platelets. Thromboxane $\mathrm{A}_{2}$ provides the prothombotic arm of platelet vascular homeostasis and is counterbalanced by the endothelial production of prostacyclin $\left(\mathrm{PGI}_{2}\right)$ which is antithrombotic.

The balance of these mediators is such that adverse thrombotic events generally constitute a greater hazard than uncontrolled bleeding. This is why inhibition of platelet COX-1 by low dose aspirin has a generally desirable effect when given to the elderly and others at risk of thrombosis. COX-2 inhibitors do not inhibit platelets and therefore do not have the antiplatelet effect of low dose aspirin. This lack of antithrombotic protection may be compounded by the inhibition of vascular prostacyclin production by selective COX-2 inhibitors. ${ }^{2}$ This latter effect 
was shown recently and supports evidence that prostacyclin synthesis is COX-2 dependent. ${ }^{3}$

Given available evidence and uncertainties, what provisional advice should be given regarding the selective COX-2 inhibitors in the symptomatic treatment of osteoarthritis? On the grounds of cost alone, paracetamol should remain the drug of first choice for those in whom it provides worthwhile symptomatic relief. Patients for whom NSAIDs could provide significant relief, were it not for unacceptable adverse effects or the risk of upper gastrointestinal events, stand to benefit most from COX-2 inhibitors. However, 500 low-risk patients may need to be treated with a COX-2 inhibitor instead of an NSAID to prevent one complicated ulcer. ${ }^{4}$ It has not yet been resolved whether age itself constitutes a risk of upper gastrointestinal events which is large enough to warrant selection of a COX-2 inhibitor instead of a conventional NSAID.

However, it should be noted that low dose aspirin should be continued where it is indicated and particularly so when a selective COX-2 inhibitor is being used (see above). Since the known variability between individuals in responsiveness to particular NSAIDs seems to extend to COX-2 inhibitors, patients who are changed from an NSAID that gives relief to a COX-2 inhibitor may be disappointed. The advantage of reduced risk for a seemingly remote contingency may be associated with less complete control of symptoms.

With regard to the inflammatory arthropathies, it should be noted that management of the prototypic disorder, rheumatoid arthritis, has changed considerably. ${ }^{5}$ In recent onset polyarthritis, there is an impetus for early intervention with multiple therapies in patients at risk for ongoing disease and a poor prognosis. While definitive therapeutic strategies are yet to be determined, combinations such as methotrexate (with a modest folate supplement), sulfasalazine and hydroxychloroquine seem to hold most promise. ${ }^{6}$ NSAIDs, including the COX-2 inhibitors, have a marginal role in these protocols as they may reduce symptoms without improving long-term outcomes, while displacing potentially more effective longer-acting interventions. The early use of prednisolone (or other glucocorticoids) is generally unhelpful, as it confounds clinical assessment and, through its endocrine action, is associated with inevitable unwanted effects. The place of newer biological therapies such as etanercept has not yet been resolved. On the grounds of cost alone, they are likely to be restricted to patients who do not respond to conventional long-acting drugs.

\section{REFERENCES}

1. Ettinger WH Jr, Burns R, Messier SP, Applegate W, Rejeski WJ, Morgan $\mathrm{T}$, et al. A randomized trial comparing aerobic exercise and resistance exercise with a health education program in older adults with knee osteoarthritis. The Fitness Arthritis and Seniors Trial. JAMA 1997;277:25-31.

2. McAdam BF, Catella-Lawson F, Mardini IA, Kapoor S, Lawson JA, FitzGerald GA. Systemic biosynthesis of prostacyclin by cyclooxygenase (COX)-2: the human pharmacology of a selective inhibitor of COX-2. Proc Natl Acad Sci USA 1999;96:272-7.

3. Topper JN, Cai J, Falb D, Gimbrone MA Jr. Identification of vascular endothelial genes differentially responsive to fluid mechanical stimuli: cyclooxygenase-2, manganese superoxide dismutase, and endothelial cell nitric oxide synthase are selectively up-regulated by steady laminar shear stress. Proc Natl Acad Sci USA 1996;93:10417-22.

4. Peterson WL, Cryer B. COX-1-sparing NSAIDS - Is the enthusiasm justified? JAMA 1999;282:1961-3.

5. Egsmose C, Lund B, Borg G, Pettersson H, Berg E, Brodin U, et al. Patients with rheumatoid arthritis benefit from early 2 nd line therapy: 5 year followup of a prospective double blind placebo controlled trial. J Rheumatol 1995;22:2208-13.

6. O'Dell JR, Haire CE, Erikson N, Drymalski W, Palmer W, Eckhoff PJ, et al. Treatment of rheumatoid arthritis with methotrexate alone, sulfasalazine and hydroxychloroquine, or a combination of all three medications. N Engl J Med 1996;334:1287-91.

\section{Letters}

Letters, which may not necessarily be published in full, should be restricted to not more than 250 words. When relevant, comment on the letter is sought from the author. Due to production schedules, it is normally not possible to publish letters received in response to material appearing in a particular issue earlier than the second or third subsequent issue.

\section{Rifampicin and contraception}

Editor, - The article 'Common questions about the management of meningococcal disease' (Aust Prescr $1999 ; 22: 117-8)$ discusses the efficacy of oral contraception following chemoprophylaxis for contacts of meningococcal disease. I have discussed this issue with the Family Planning Association and believe in-depth advice on how to manage contraception while taking rifampicin should be given to the contact.

Appropriate advice is: 'In the case of short term concurrent drug treatment, a barrier method should be used both during treatment and for seven days after discontinuation. If this would continue into the next oral contraceptive tablet-free interval, the woman should skip the tablet-free interval and start the next pack as soon as she has finished the pack in use.' This is an important issue, as advising women to stop oral contraception or use another method for four weeks after completion of chemoprophylaxis, increases the risk of non-compliance and causes further stress to the contact. It is also excessive and not necessary.

Giulietta Pontivivo

Registered Nurse

South East Sydney Public Health Unit

Sydney, NSW

\section{REFERENCE}

1. Geurts TBP, Goorissen EM, Sitsen JMA. Summary of drug interactions with oral contraceptives. Carnforth: Parthenon Publishing Group; 1993. p. 72-3. 